\title{
A COMBINATORIAL FORMULA FOR THE NORMAL EULER CLASS OF A LATTICE 2-MANIFOLD IN 4-SPACE
}

\author{
BORIS V. YUSIN
}

\begin{abstract}
For a lattice 2-manifold immersed in 4-space with all faces parallel to coordinate planes it is possible to assign a local index to each vertex type in a unique way so that the sum of vertex indices gives the normal Euler number of the immersion. Related results for simplicial immersions have been established by $\mathrm{T}$. Banchoff.
\end{abstract}

1. Introduction. The normal Euler number for a smooth immersion $f: M^{2 n} \rightarrow \mathbf{R}^{4 n}$ is given by the algebraic number of self-intersection points of $f$, multiplied by -2 , provided that $M^{2 n}$ is smooth, closed and orientable. In the case where $f$ is a polyhedral immersion, Banchoff [1] has established the existence of a local combinatorial formula for the normal Euler number $\nu(f)$; he has indicated also a procedure for obtaining an explicit form of such a formula. Here we consider the case when a 2-manifold is immersed in $\mathbf{R}^{4}$ with all faces parallel to the coordinate planes (a lattice 2-manifold in $\mathbf{R}^{4}$ ). We shall obtain the explicit formula for $\nu(f)$ by listing all possible vertex types, and shall prove the uniqueness of the formula in our case.

2. Statement of the main result. Let $f: M^{2} \rightarrow \mathbf{R}^{4}$ be a lattice manifold with $M^{2}$ orientable. We shall assume that the immersion $f$ is generic in the sense that the only self-intersections occur at isolated points of $M^{2}$.

By the type of a lattice manifold at a vertex $A$ in $M^{2}$ we mean its tangent cone at $f(A)$, i.e. the cone in $\mathbf{R}^{4}$, containing the image of the star of $A$ in $M^{2}$. We denote this type by $f_{A}$.

Since each vertex type is a union of angles in $\mathbf{R}^{4}$, spanned by pairs of unit vectors in any direction along coordinate axes, there are only finitely many such types. The group $G$ of symmetries of the lattice, generated by permutations of axes and reflections in hyperplanes, acts on this collection of types.

THEOREM. There is a function on the set of vertex types such that the sum of its values at the vertices of $M^{2}$ is equal to $\nu(f)$. There exists a single such function $\nu$ satisfying the condition of $G$-alternating, i.e. such that $\nu\left(\pi f_{A}\right)= \pm \nu\left(f_{A}\right)$ depending on whether the isometry $\pi$ preserves or reverses orientation. This function determines the local combinatorial formula.

Received by the editors December 29, 1979 and, in revised form, August 12, 1983.

1980 Mathematics Subject Classification. Primary 57R20, 57Q35, 53C42; Secondary 52A25.

Key words and phrases. Normal curvature, normal Euler class, lattice 2-manifold, curvature for polyhedra, combinatorial formulas for characteristic classes. 
The existence of such a formula directly follows from the more general result proved by Banchoff [1]. In the next section we shall exhibit a complete set of $G$-inequivalent vertex types and for each of them we shall find the value at this vertex type of the function mentioned in the Theorem (the index of this type). This will prove the uniqueness of the combinatorial formula.

3. Computation of the indices. There are 23 vertex types which are inequivalent under the action of $G$. We label them by giving a sequence of unit vectors $\pm 1, \pm 2, \pm 3, \pm 4$, in a positive or negative direction along the four coordinate axes. Note that if we can find in the group $G$ an odd transformation which fixes a given vertex type, then the index of this type is zero. Thus the index of any vertex type which lies in a Euclidean 3-subspace must be zero, because the reflection in this subspace fixes it.

There are 6 vertex types which lie in a Euclidean 3-subspace:

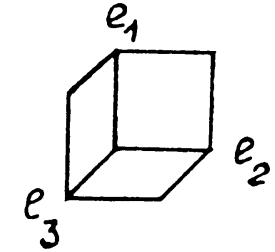

$$
\nu(1,2,3)=0
$$

Figure 1

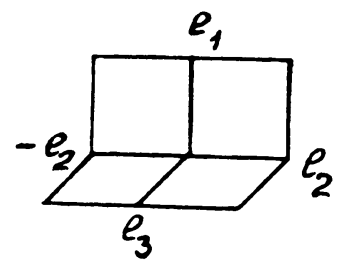

$$
\nu(1,2,3,-2)=0
$$

Figure 3

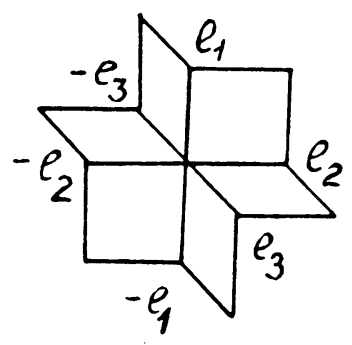

$$
\nu(1,2,3,-1,-2,-3)=0
$$

FIGURE 5

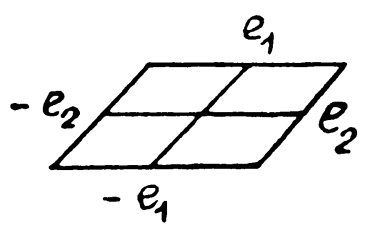

$$
v(1,2,-1,-2)=0
$$

FIGURE 2

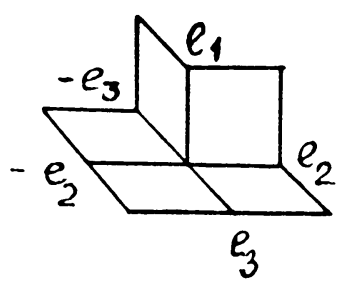

$$
\nu(1,2,3,-2,-3)=0
$$

FIGURE 4

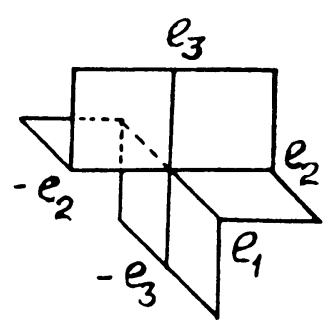

$\nu(1,2,3,-2,-1,-3)=0$

Figure 6 
Among the other types there are 5, also admitting odd isometry:

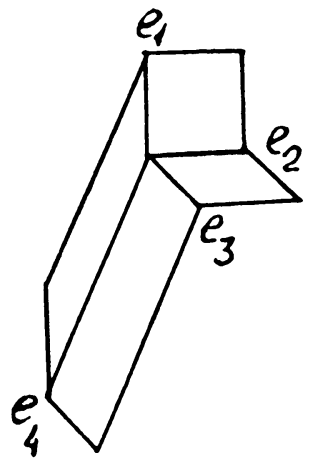

$$
\begin{aligned}
& \nu(1,2,3,4)=0, \\
& \text { isometry }\left(\begin{array}{llll}
1 & 2 & 3 & 4 \\
2 & 3 & 4 & 1
\end{array}\right)
\end{aligned}
$$

(cyclic permutation of axes)

\section{FIGURE 7}

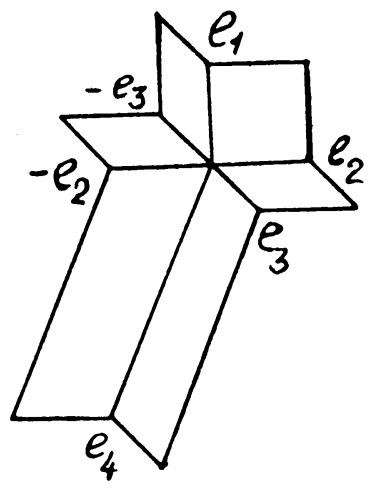

$$
\begin{aligned}
& v(1,2,3,4,-2,-3)=0 \\
& \text { isometry }\left(\begin{array}{rrrr}
1 & 2 & 3 & 4 \\
1 & -3 & -2 & 4
\end{array}\right)
\end{aligned}
$$

(reflection in the hyperplane with $e_{2}+e_{3}$ as normal)

Figure 8

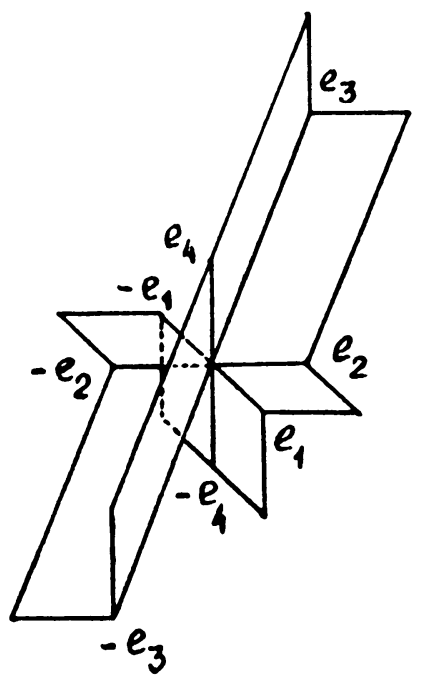

$$
\begin{aligned}
& \nu(1,2,3,4,-3,-2,-1,-4)=0 \\
& \text { isometry }\left(\begin{array}{rrrr}
1 & 2 & 3 & 4 \\
-3 & -2 & -1 & -4
\end{array}\right)
\end{aligned}
$$

FigURE 9 


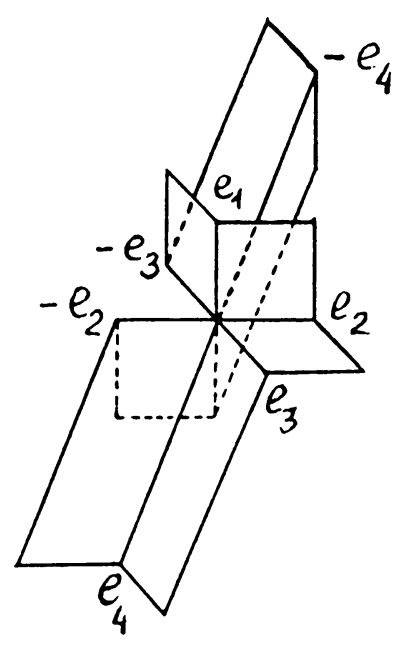

$$
\begin{aligned}
& \nu(1,2,3,4,-2,-1,-4,-3)=0 \\
& \text { isometry }\left(\begin{array}{rrrr}
1 & 2 & 3 & 4 \\
-4 & -3 & 1 & 2
\end{array}\right)
\end{aligned}
$$

Figure 10

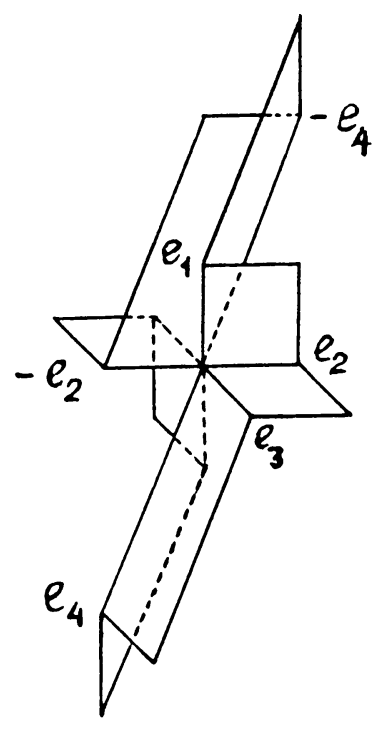

$$
\begin{aligned}
& \nu(1,2,3,4,-1,-3,-2,-4)=0 \\
& \text { isometry }\left(\begin{array}{rrrr}
1 & 2 & 3 & 4 \\
-1 & -3 & -2 & -4
\end{array}\right)
\end{aligned}
$$

\section{Figure 11}

The remaining 12 types have nonzero indices. They are shown in Figures $12 \mathrm{~d}$ and $13 b-23 b$. We shall find the values of their indices by exhibiting some lattice polyhedra. The first of them has a single self-intersection point. Topologically it is an immersion of the 2-sphere, consisting of two orthogonal intersection disks and a homotopy between their boundaries. The homotopy lies in the 3-sphere around the intersection point. In order to make this immersion a lattice polyhedron, we change disks for $2 \times 2$ squares, intersecting each other at their centers, and change the 3 -sphere for the boundary of the $2 \times 2 \times 2 \times 2$ cube. The polyhedron consists of two parts, each of them lying in a 3-subspace. The parts are shown in Figures 12a and $12 \mathrm{~b}$; they are "glued together" along the line which is shown as a double line on each figure. The corresponding cell decomposition of the 2-sphere is shown in Figure $12 \mathrm{c}$; for each vertex coordinates of its image are also indicated there. 

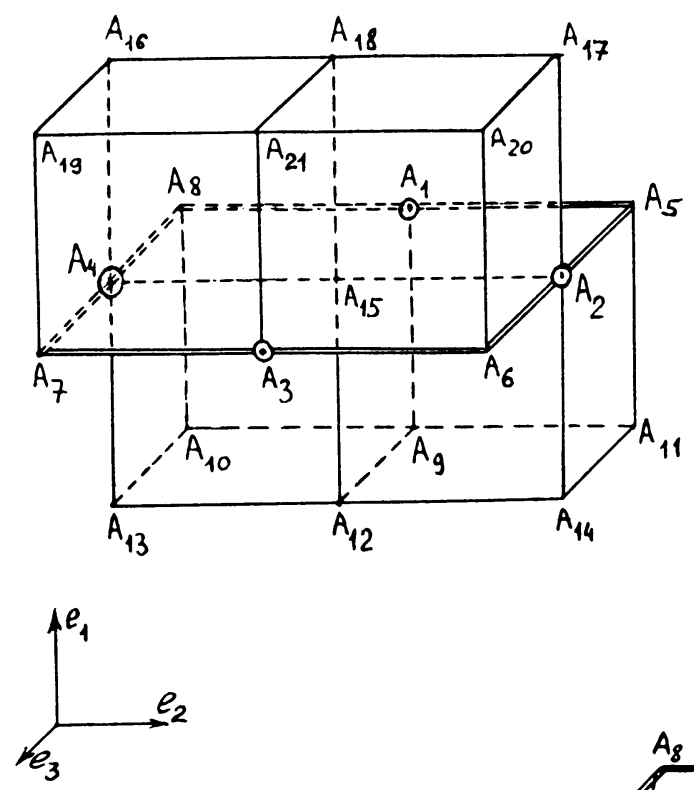

Figure 12a

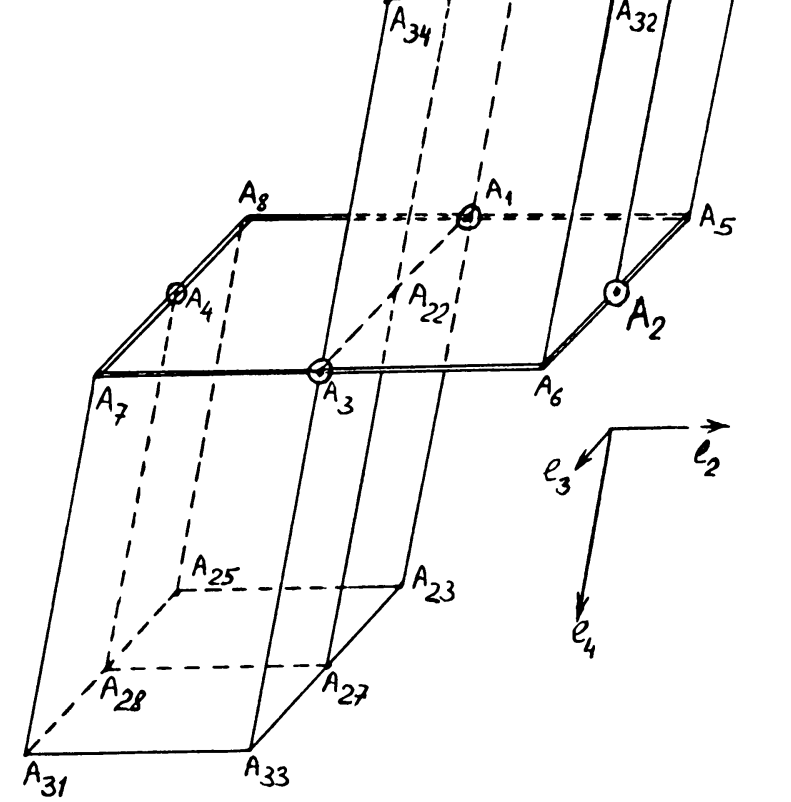

FIGURE 12b

What does our combinatorial formula mean for this lattice polyhedron? Since types of vertices inside each part of the polyhedron lie in the 3-subspace containing the entire part, all these vertices must have zero indices. So only eight vertices on the "gluing line" may have nonzero indices. But four of them $\left(A_{5}, A_{6}, A_{7}, A_{8}\right)$ are of the type shown in Figure 7 and therefore also have zero indices. The vertex type of each of the remaining four vertices (marked by a small circle around each of them) can be transformed by an odd isometry into the type shown in Figure 12d. These

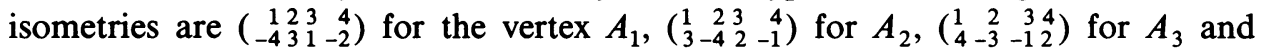

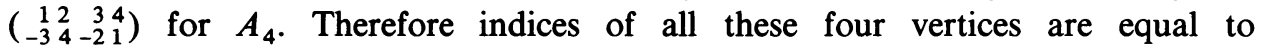
$-\nu(1,2,3,4,-3,-2)$. Since the normal Euler number of the surface is -2 (it has a single self-intersection point with index +1 ), indices of $A_{1}, A_{2}, A_{3}, A_{4}$ are equal to $-1 / 2$ and $\nu(1,2,3,4,-3,-2)=1 / 2$. 


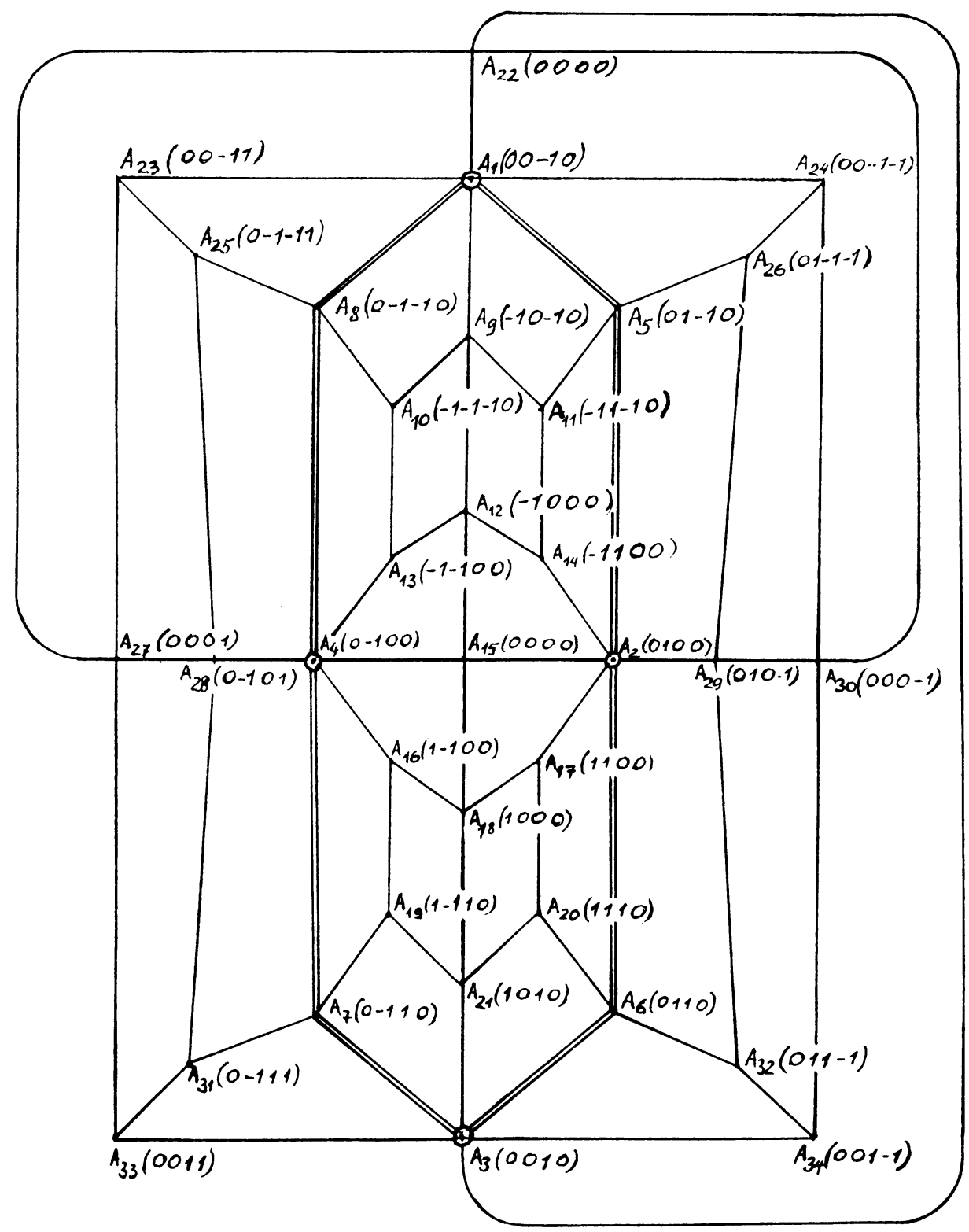

FIGURE 12c

After we have found the index of one vertex type with nonzero index, the indices of the other types may be found by exhibiting them on surfaces without self-intersections. These surfaces are shown in Figures 13a-23a. Each of them has only 2 or 3 vertices, marked $A_{1}, A_{2}, A_{3}$, such that their types do not lie in a Euclidean 3-subspace and are inequivalent to the one shown in Figure 7; these vertices are also marked by a small circle around each of them. Only these vertices may have 


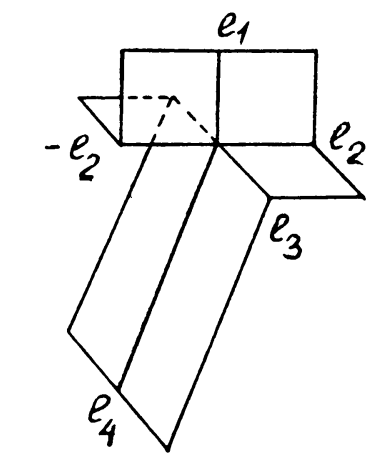

$$
\nu(1,2,3,4,-3,-2)=1 / 2
$$

Figure 12d

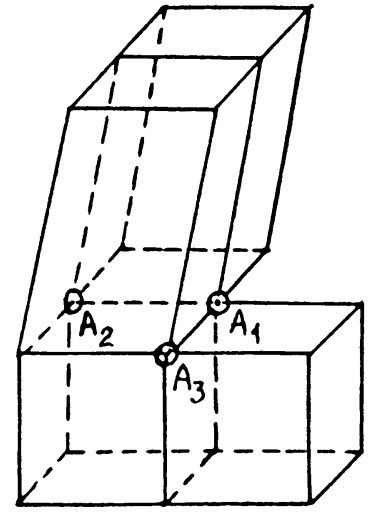

Figure 13a

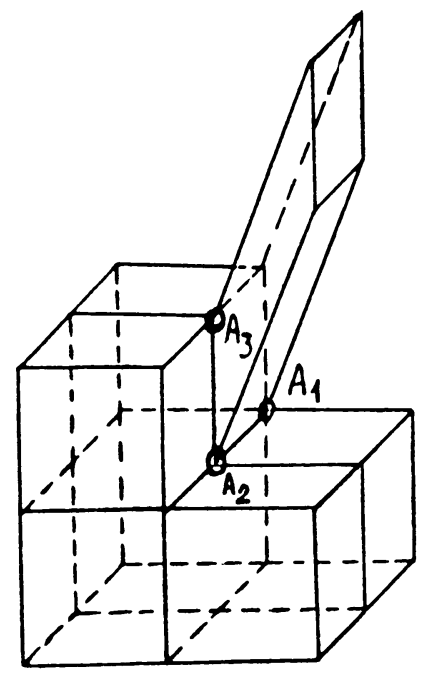

Figure 14a

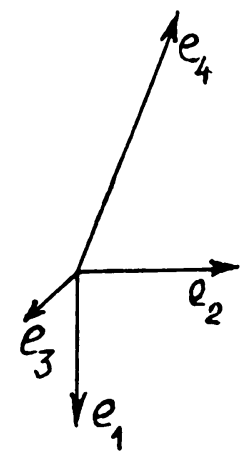

$v(1,2,3,4,-1,-2)=1 / 2$

Figure 14b 


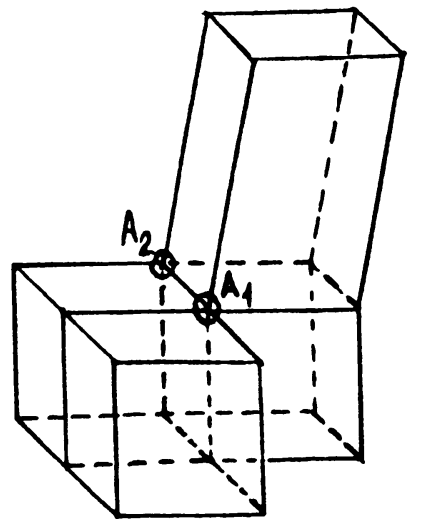

FIGURE 15a
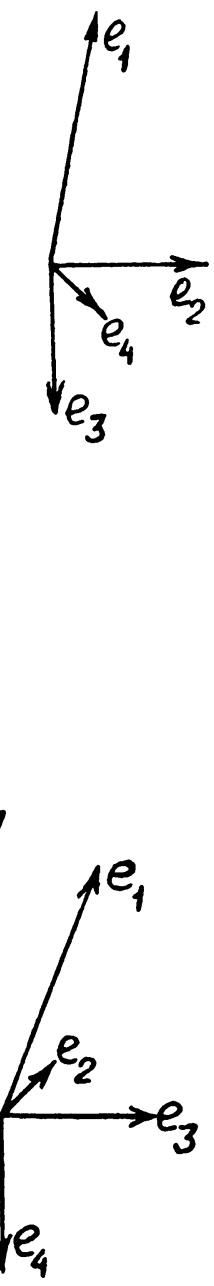

Figure 16a

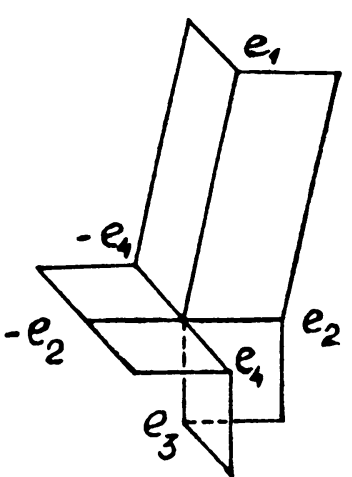

$\nu(1,2,3,4,-2,-4)=1 / 4$

FIGURE 15b

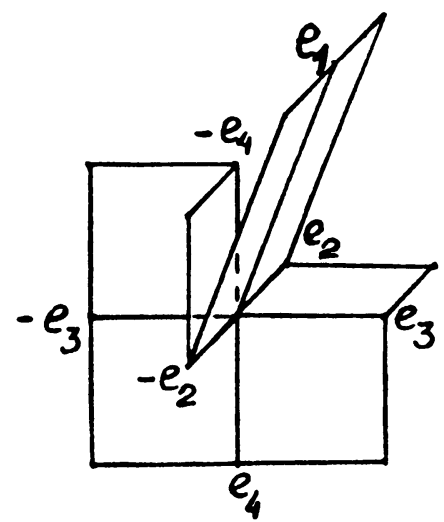

$\nu(1,2,3,4,-3,-4,-2)=3 / 4$

nonzero indices. Since the normal Euler number of each of these surfaces is zero, we can find value of all the indices. Thus, in Figure 13a

$$
\nu\left(f_{A_{1}}\right)=\nu(1,2,3,4,-3,-2)=1 / 2 \text {; }
$$

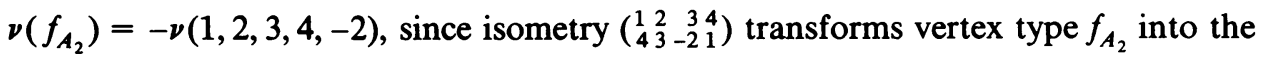
one shown in Figure 13b;

$$
\nu\left(f_{A_{3}}\right)=-\nu(1,2,3,4,-2) \quad\left(\text { isometry }\left(\begin{array}{cccc}
1 & 2 & 3 & 4 \\
1 & 2 & -3 & 4
\end{array}\right)\right),
$$

therefore $\nu(1,2,3,4,-2)=1 / 4$ (Figure 13b). 


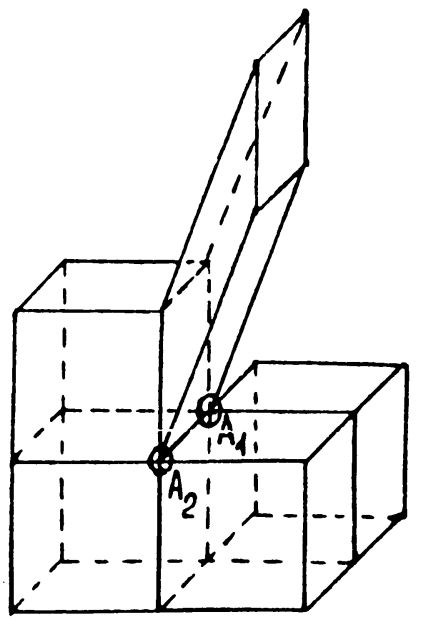

FIGURE 17a

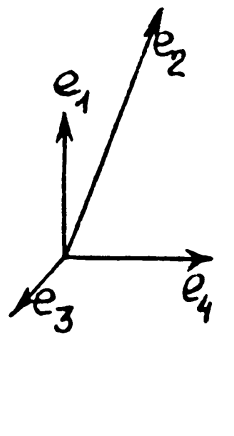

$\nu(1,2,3,4,-3,-1,-4)=1 / 2$

FIGURE 17b

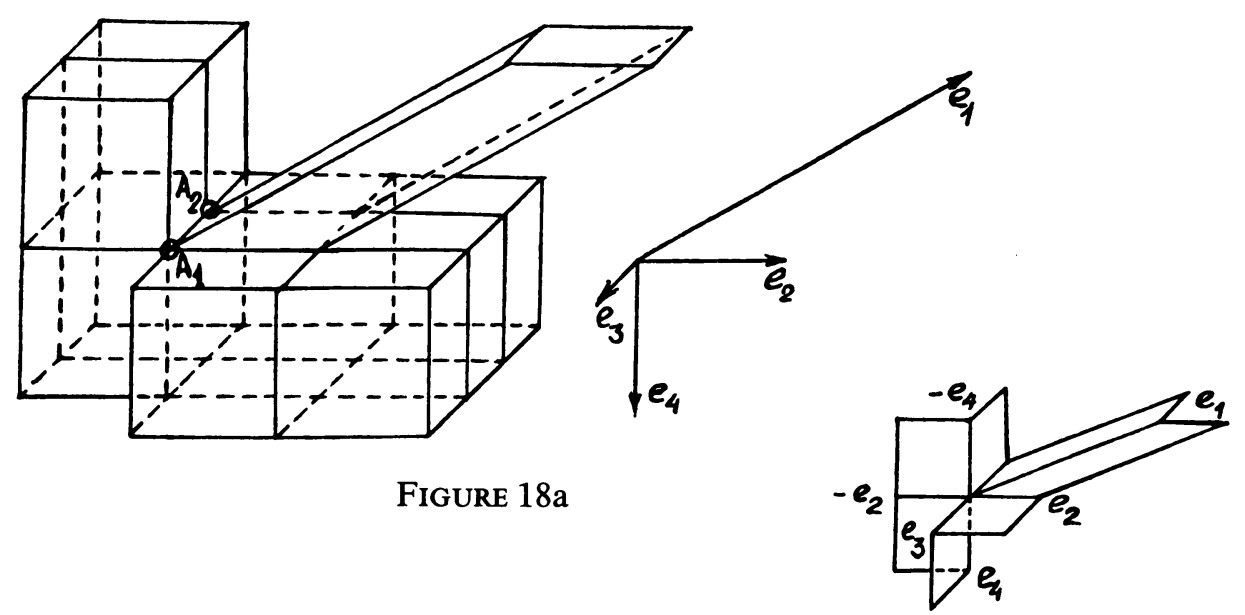

$$
\nu(1,2,3,4,-2,-4,-3)=-1 / 4
$$

FIGURE 18b

In Figure 14a,

$$
\begin{array}{ll}
\nu\left(f_{A_{1}}\right)=\nu(1,2,3,4,-1,-2), & \\
\nu\left(f_{A_{2}}\right)=-\nu(1,2,3,4,-2) & \left(\text { isometry }\left(\begin{array}{cccc}
1 & 2 & 3 & 4 \\
-3 & 1 & 2 & 4
\end{array}\right)\right), \\
\nu\left(f_{A_{3}}\right)=-\nu(1,2,3,4,-2) & \left(\text { isometry }\left(\begin{array}{cccc}
1 & 2 & 3 & 4 \\
3 & -1 & 2 & 4
\end{array}\right)\right),
\end{array}
$$

therefore $\nu(1,2,3,4,-1,-2)=1 / 2$ (Figure 14b). 
In Figure 15a,

$$
\begin{aligned}
& \nu\left(f_{A_{1}}\right)=\nu(1,2,3,4,-2,-4), \\
& \nu\left(f_{A_{2}}\right)=-\nu(1,2,3,4,-2) \quad\left(\text { isometry }\left(\begin{array}{cccc}
1 & 2 & 3 & 4 \\
4 & -2 & 1 & 3
\end{array}\right)\right),
\end{aligned}
$$

therefore $\nu(1,2,3,4,-2,-4)=1 / 4$ (Figure 15b).

In Figure 16a,

$$
\begin{aligned}
& \nu\left(f_{A_{1}}\right)=\nu(1,2,3,4,-3,-4,-2), \\
& \nu\left(f_{A_{2}}\right)=-\nu(1,2,3,4,-1,-2) \quad\left(\text { isometry }\left(\begin{array}{llll}
1 & 2 & 3 & 4 \\
3 & 4 & 2 & 1
\end{array}\right)\right), \\
& \nu\left(f_{A_{3}}\right)=-\nu(1,2,3,4,-2) \quad\left(\text { isometry }\left(\begin{array}{cccc}
1 & 2 & 3 & 4 \\
1 & 2 & -3 & 4
\end{array}\right)\right),
\end{aligned}
$$

therefore $\nu(1,2,3,4,-3,-4,-2)=3 / 4$ (Figure 16b).

In Figure 17a,

$$
\begin{aligned}
& \nu\left(f_{A_{1}}\right)=\nu(1,2,3,4,-3,-1,-4), \\
& \nu\left(f_{A_{2}}\right)=-\nu(1,2,3,4,-1,-2) \quad\left(\text { isometry }\left(\begin{array}{cccc}
1 & 2 & 3 & 4 \\
2 & 3 & -4 & -1
\end{array}\right)\right),
\end{aligned}
$$

therefore $\nu(1,2,3,4,-3,-1,-4)=1 / 2$ (Figure 17b).

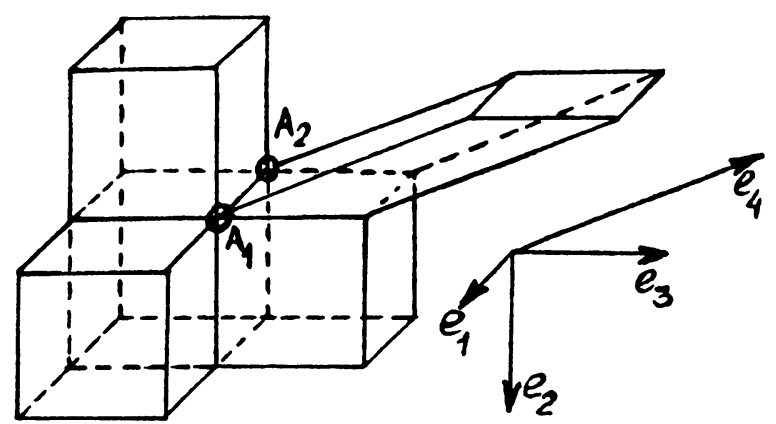

FIGURE 19a

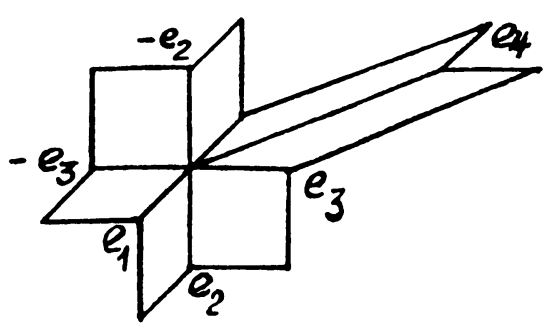

$$
\nu(1,2,3,4,-1,-2,-3)=1 / 2
$$

Figure 19b 


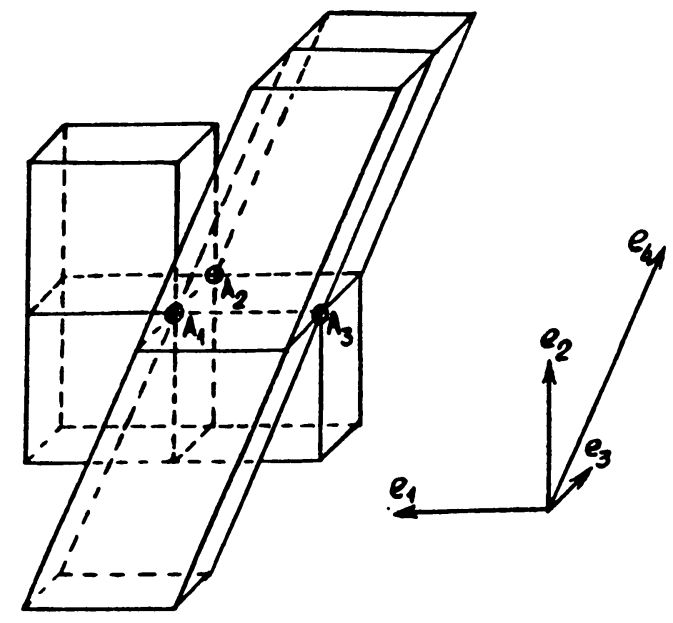

FIGURE 20a

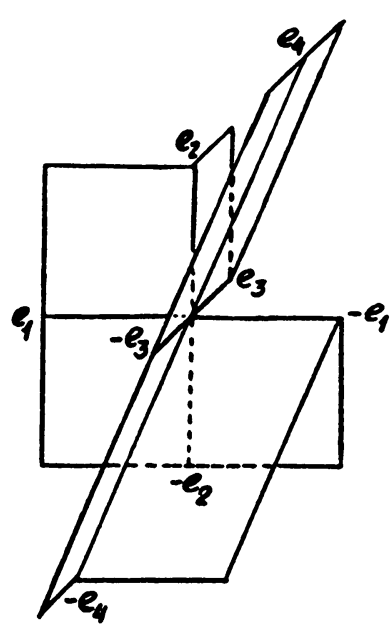

$v(1,2,3,4,-3,-4,-1,-2)=1$

FigURE 20b

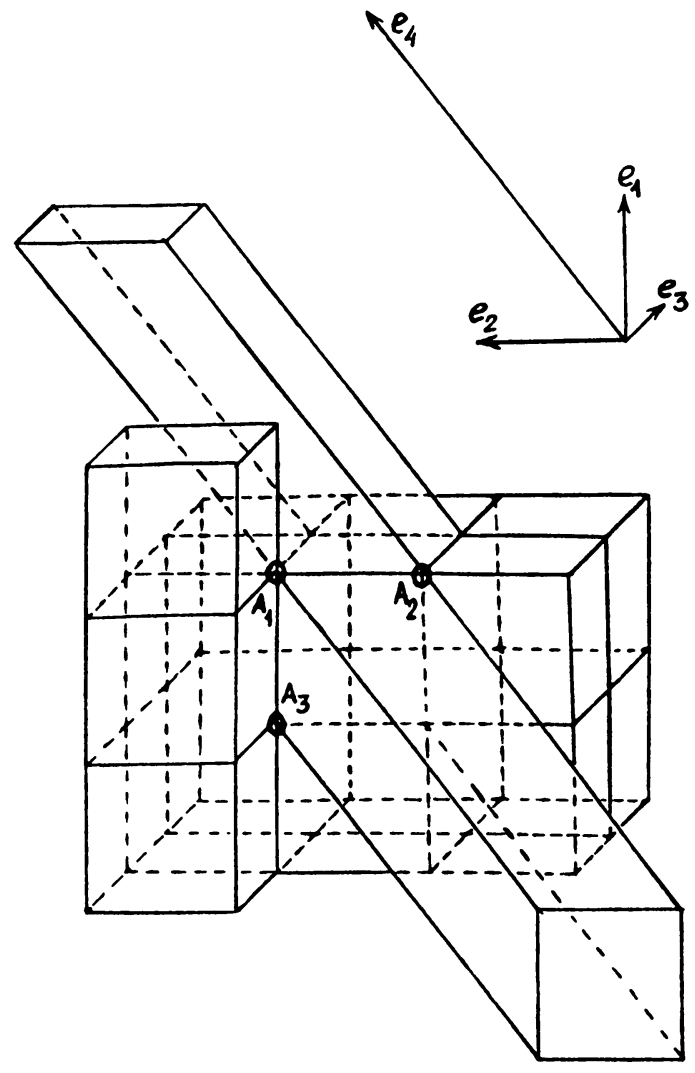

FIGURE 21a 


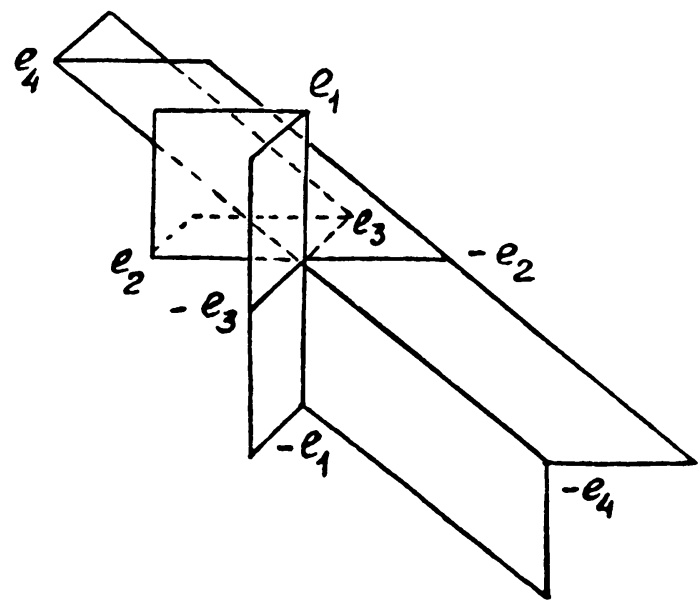

$$
\nu(1,2,3,4,-2,-4,-1,-3)=-1 / 2
$$

FIGURE 21b

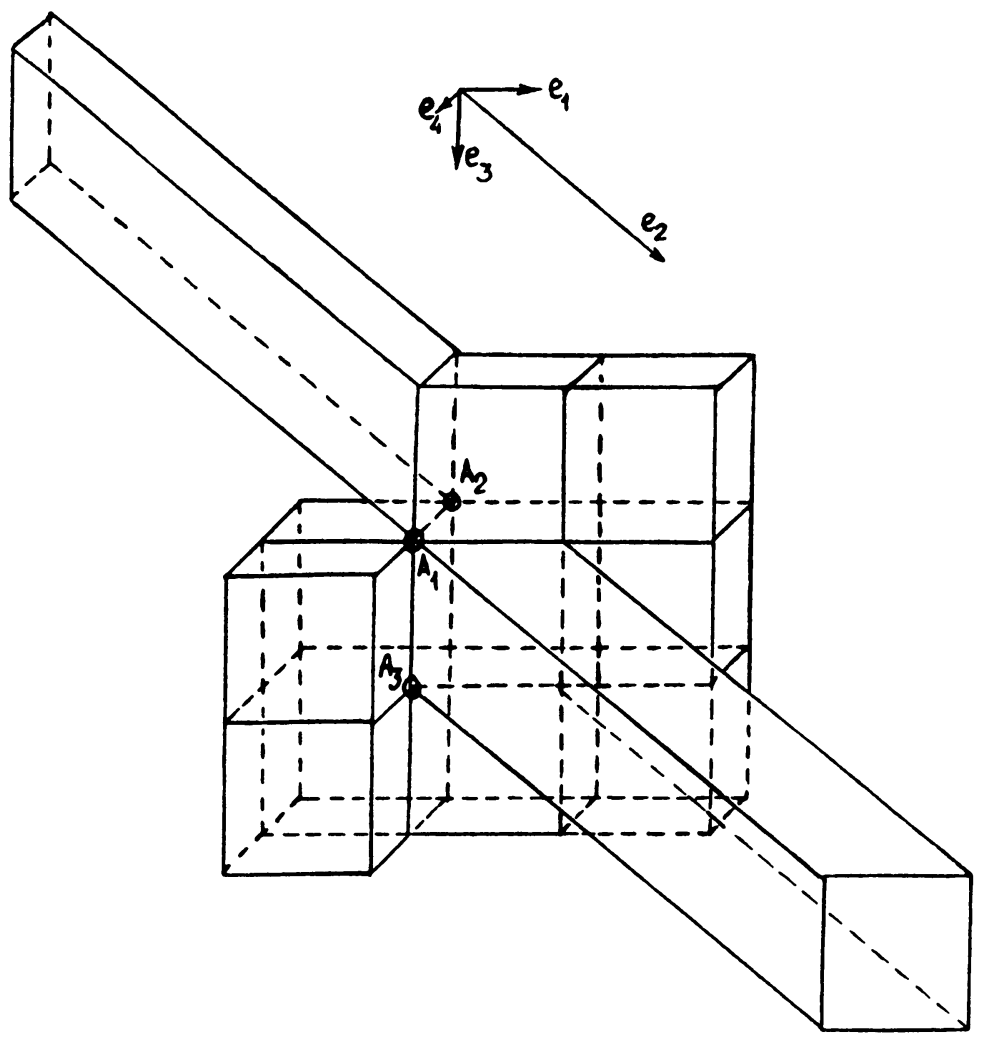

Figure 22a 


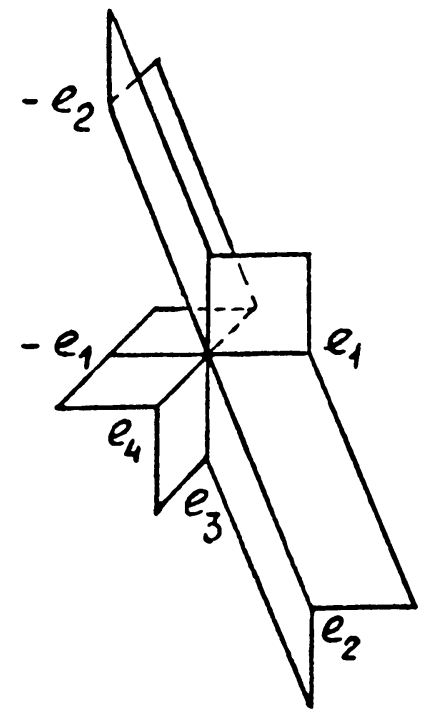

$$
\nu(1,2,3,4,-1,-4,-2,-3)=3 / 4
$$

FIGURE 22b

In Figure 18a,

$$
\begin{aligned}
\nu\left(f_{A_{1}}\right) & =\nu(1,2,3,4,-2,-4,-3), \\
\nu\left(f_{A_{2}}\right) & =\nu(1,2,3,4,-2) \quad\left(\text { isometry }\left(\begin{array}{cccc}
1 & 2 & 3 & 4 \\
3 & 4 & 2 & -1
\end{array}\right)\right),
\end{aligned}
$$

therefore $\nu(1,2,3,4,-2,-4,-3)=-1 / 4$ (Figure 18b).

In Figure 19a,

$$
\begin{aligned}
& \nu\left(f_{A_{1}}\right)=\nu(1,2,3,4,-1,-2,-3), \\
& \nu\left(f_{A_{2}}\right)=-\nu(1,2,3,4,-1,-2) \quad\left(\text { isometry }\left(\begin{array}{llll}
1 & 2 & 3 & 4 \\
4 & 1 & 2 & 3
\end{array}\right)\right),
\end{aligned}
$$

therefore $\nu(1,2,3,4,-1,-2,-3)=1 / 2$ (Figure 19b).

In Figure 20a,

$$
\begin{array}{ll}
\nu\left(f_{A_{1}}\right)=\nu(1,2,3,4,-3,-4,-1,-2), & \\
\nu\left(f_{A_{2}}\right)=-\nu(1,2,3,4,-1,-2) & \left(\text { isometry }\left(\begin{array}{cccc}
1 & 2 & 3 & 4 \\
1 & 2 & -3 & 4
\end{array}\right)\right), \\
\nu\left(f_{A_{3}}\right)=-\nu(1,2,3,4,-1,-2) & \left(\text { isometry }\left(\begin{array}{cccc}
1 & 2 & 3 & 4 \\
4 & -3 & 2 & 1
\end{array}\right)\right),
\end{array}
$$

therefore $\nu(1,2,3,4,-3,-4,-1,-2)=1$ (Figure 20b). 


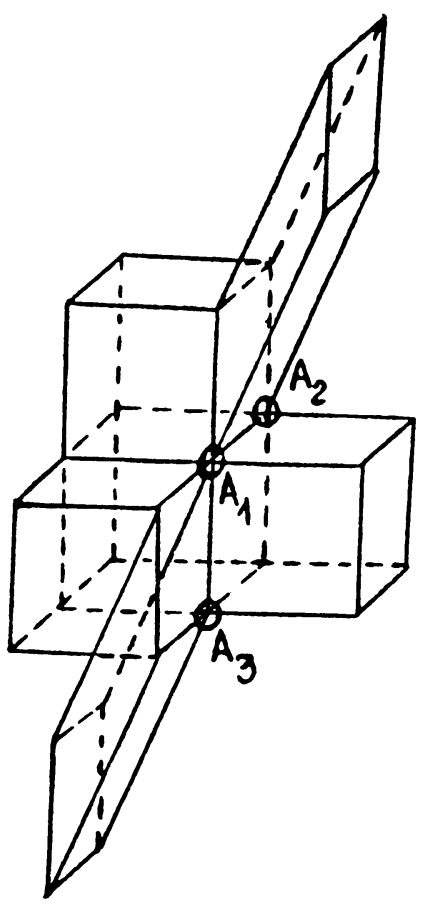

Figure 23a

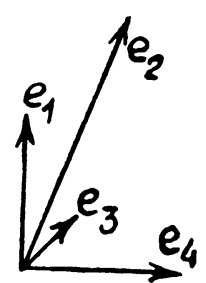

$\nu(1,2,3,4,-1,-2,-3,-4)=1$

Figure 23b

In Figure 21a,

$$
\begin{aligned}
& \nu\left(f_{A_{1}}\right)=\nu(1,2,3,4,-2,-4,-1,-3), \\
& \nu\left(f_{A_{2}}\right)=\nu(1,2,3,4,-2,-4) \quad\left(\text { isometry }\left(\begin{array}{cccc}
1 & 2 & 3 & 4 \\
-1 & -2 & 3 & 4
\end{array}\right)\right), \\
& \nu\left(f_{A_{3}}\right)=\nu(1,2,3,4,-2) \quad\left(\text { isometry }\left(\begin{array}{cccc}
1 & 2 & 3 & 4 \\
2 & -4 & -1 & -3
\end{array}\right)\right),
\end{aligned}
$$

therefore $\nu(1,2,3,4,-2,-4,-1,-3)=-1 / 2$ (Figure 21b).

In Figure 22a,

$$
\begin{aligned}
& \nu\left(f_{A_{1}}\right)=\nu(1,2,3,4,-1,-4,-2,-3), \\
& \nu\left(f_{A_{2}}\right)=-\nu(1,2,3,4,-1,-2) \quad\left(\text { isometry }\left(\begin{array}{cccc}
1 & 2 & 3 & 4 \\
-2 & -4 & 1 & 3
\end{array}\right)\right), \\
& \nu\left(f_{A_{3}}\right)=-\nu(1,2,3,4,-2) \quad\left(\text { isometry }\left(\begin{array}{cccc}
1 & 2 & 3 & 4 \\
3 & 4 & 2 & 1
\end{array}\right)\right),
\end{aligned}
$$

therefore $\nu(1,2,3,4,-1,-4,-2,-3)=3 / 4$ (Figure 22b).

In Figure 23a,

$$
\begin{aligned}
& \nu\left(f_{A_{1}}\right)=\nu(1,2,3,4,-1,-2,-3,-4), \\
& \nu\left(f_{A_{2}}\right)=-\nu(1,2,3,4,-1,-2) \quad\left(\text { isometry }\left(\begin{array}{cccc}
1 & 2 & 3 & 4 \\
2 & 3 & -4 & -1
\end{array}\right)\right),
\end{aligned}
$$




$$
\nu\left(f_{A_{3}}\right)=-\nu(1,2,3,4,-1,-2) \quad\left(\text { isometry }\left(\begin{array}{cccc}
1 & 2 & 3 & 4 \\
4 & -3 & -2 & -1
\end{array}\right)\right),
$$

therefore $\nu(1,2,3,4,-1,-2,-3,-4)=1$ (Figure 23b).

To finish the computation of the indices and to prove the uniqueness of the combinatorial formula, we only need to know that our list of vertex types is complete.

Proposition. Any other vertex type can be obtained from the types shown in Figures 1-23 by lattice isometries.

Proof. Consider any vertex type which does not lie in any Euclidean 3-subspace. One can see that in the corresponding sequence of unit vectors four successive independent vectors can be found, which may be transformed into the sequence $e_{1}, e_{2}, e_{3}, e_{4}$, i.e. $1,2,3,4$. Then the whole sequence will be transformed into a sequence of one of the following forms (depending on its length): 1, 2, 3, 4, $-a,-b,-c,-d ; 1,2,3,4,-a,-b,-c ; 1,2,3,4,-a,-b ; 1,2,3,4,-a ; 1,2,3,4$, where $a, b, c, d$ is a permutation of $1,2,3,4$. The number of such sequences is not too large and it is possible to examine each of them, but we omit this examination. The more simple case of a vertex type lying in a Euclidean 3-subspace is quite similar and we also omit it.

The author is glad to express his gratitude to Professor D. B. Fuks who has rendered him great help in his work, to Professor Thomas Banchoff for his suggestions for the improvement of the paper, and to Professor Robert MacPherson for helpful discussion.

\section{REFERENCES}

1. T. Banchoff, Normal curvatures and Euler classes for polyhedral surfaces in 4-space, Proc. Amer. Math. Soc. 92 (1984), 593-596. 